\title{
Hands on CRESST: Determining the energy dependence of the $\alpha$ band in $\mathrm{CaWO}_{4}$
}

Julieta Gruszko* Center for Experimental Nuclear Physics and Astrophysics, University of Washington

E-mail: jgruszko@uw. edu

\section{Wei Zhao}

Key Laboratory of Particle and Radiation Imaging (Ministry of Education) and Department of Engineering Physics, Tsinghua University

E-mail: seawolfzw921@gmail.com

\section{Karoline Schäffner}

INFN - Laboratori Nazionali del Gran Sasso

E-mail: karoline.schaeffneralngs.infn.it

The energy dependence of light yield due to $\alpha$ particle interactions in scintillating $\mathrm{CaWO}_{4}$ crystals was studied. As seen in a previous measurement, these crystals, used in CRESST-II, exhibit higher than expected light yields at low energies. This has significant implications for WIMP search background models. The setup of this measurement, using the CRESST test cryostat at LNGS, is described, and preliminary data are presented.

Gran Sasso Summer Institute 2014 Hands-On Experimental Underground Physics at LNGS 22 September - 03 October 2014

INFN - Laboratori Nazionali del Gran Sasso, Assergi, Italy

\footnotetext{
* Speaker.
} 


\section{Introduction}

A variety of astrophysical phenomena provide strong evidence that non-luminous matter makes up roughly $85 \%$ of the matter in the universe. A well-supported candidate among the many theoretical models proposed for dark matter, Weakly Interacting Massive Particles (WIMPs) are commonly searched for via their elastic scattering off of target nuclei.

The CRESST-II experiment aims to observe this interaction in scintillating $\mathrm{CaWO}_{4}$ crystals operated at $\mathrm{mK}$ temperatures. Particles interacting with the crystal lattice produce both phonons, measurably raising the crystal temperature, and photons, via scintillation. Most of the incoming particle's energy is converted to phonons, so measurements in this channel provide a sensitive and roughly particle type-independent measure of the energy. On the other hand, the light yield (the ratio of measured photon energy to phonon energy) of an interaction is highly dependent on particle type. This allows CRESST-II to quantify and reduce many of the backgrounds for WIMP-nuclear scattering, provided the light yield of various particle types is well understood.

In the CRESST analyses [1] [2], the light yield was assumed to be independent of $\alpha$ particle energy. This allowed the $\alpha$ light yield band to be extrapolated from the observed MeV-scale alpha particle interactions seen during the runs. Taking into account the finite energy resolution of the detectors, the overlap at low energies with the nuclear-recoil bands was calculated to model a possible background contribution to the WIMP signal region.

Both phenomenological models and later measurements show, however, that the $\alpha$ band is not energy independent; in fact, light yield increases at low energies. Therefore, the $\alpha$ band's overlap with the WIMP signal region is smaller than was assumed in the CRESST analyses. This implies that reliable measurements at low energies of $\alpha$ particle interactions in $\mathrm{CaWO}_{4}$ may allow CRESST to set stronger limits on WIMP interactions.

Previous measurements [3] quantified the energy dependence of the $\alpha$ light yield band in one $\mathrm{CaWO}_{4}$ crystal and one $\mathrm{CdWO}_{4}$ crystal. Since crystal properties can vary, similar measurements should be conducted on other CRESST crystals. During the LNGS 2014 Summer Institute, an $\alpha$ source measurement was begun of a CRESST-II detector unit made up of the $\mathrm{CaWO}_{4}$ crystal "Sabine" and the silicon-on-sapphire photon detector "Pierre."

\section{Experimental Setup}

\subsection{Crystal Mount}

The $\mathrm{CaWO}_{4}$ crystal used, Sabine, was first wrapped in reflecting and scintillating foil, save for the surfaces that will be exposed to the photon detector. This reduces nuclear-scattering backgrounds due to contaminated surfaces in the crystal mount. If the surfaces have ${ }^{210}$ Po contamination from ${ }^{222} \mathrm{Rn}$ exposure, energy-degraded ${ }^{206} \mathrm{~Pb}$ nuclei may scatter into the crystal. These events will appear in the signal region if the $\alpha$ simply escapes; when scintillating foil is used, though, the $\alpha$ scatter adds to the photon signal, increasing the light yield of the event and removing it from the signal region.

The crystal was then mounted in an low-background copper mount and secured with aluminumcoated bronze clamps, as seen in Figure 1a. These clamps must be tightened sufficiently to reduce backgrounds from microphonics without creating fractures in the crystal. 


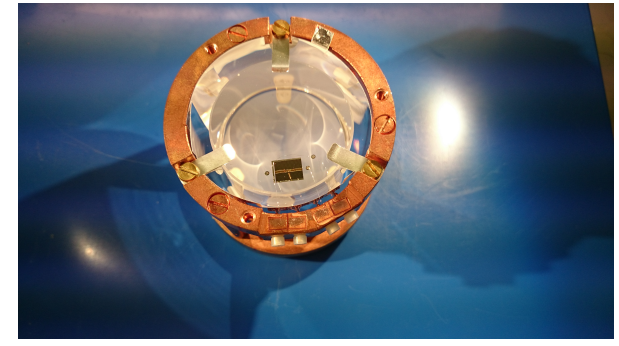

(a) The phonon detector Sabine, after being mounted. The TES, bronze clamps, and scintillating foil are visible.

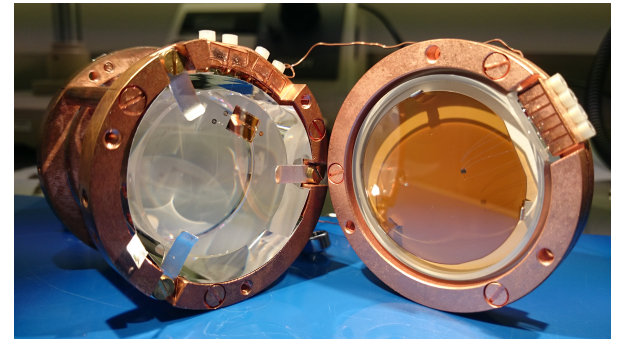

(b) Both detectors, after mounting. The two mounts are attached to one another before the detector unit is installed in the cryostat.

Figure 1: The crystal mounts used for this measurement

A similar mount, without foil, is used for the photon sensor Pierre, a silicon-on-sapphire (SOS) absorber which absorbs the blue scintillation light emitted by the $\mathrm{CaWO}_{4}$ crystal. See Figure $1 \mathrm{~b}$.

\subsection{Transition Edge Sensors (TES)}

Transition edge sensors (TES) are used as thermometers to read out the energy deposited in the crystals. The TES of the phonon detector is made of a thin tungsten film that is evaporated onto the crystal surface. This film is cooled to the temperature of its transition between superconducting and normal states. In this region, the resistance of the film is highly temperature dependent, so small temperature changes $(\mathscr{O}(\mu K))$ caused by particle interactions lead to changes measurable changes in voltage when a constant current is applied across the film. A gold structure extending across the tungsten film provides a thermal link, through which the absorber relaxes back to equilibrium after it is heated by a particle interaction. A heater, which is also thermally connected via this structure, is biased to stabilize the TES at its operating point and inject test pulses. Aluminum contact pads on the crystal surface are used to connect the bias supplies of the TES and heater via wire bonds. See Figure 1a.

Another TES measures temperature changes in the SOS absorber due to photon absorption. See Figure $1 b$.

\subsection{Sources Used}

The goal of these measurements was to measure the light yield of $\alpha$ particles over a large energy range. Therefore two $\alpha$ emitting sources were used. ${ }^{238} \mathrm{U}$, in solution, was applied to a teflon sheet. This isotope emits a $4.27 \mathrm{MeV} \alpha$. To ensure sufficient statistics at low energies, a raw piece of samarium was also used. Natural samarium has $15 \%$ abundance of ${ }^{147} \mathrm{Sm}$, which emits a $2.31 \mathrm{MeV} \alpha$. Both the teflon sheet between the source and detector, in the case of ${ }^{238} \mathrm{U}$, and the bulk geometry, in the case of ${ }^{147} \mathrm{Sm}$, degrade the $\alpha$ energy, leading to a continuum of particle energies up to $4.27 \mathrm{MeV}$. Gold foil was placed in front of the samarium to ensure that recoiling ${ }^{143} \mathrm{Nd}$ nuclei were not seen by the detector.

${ }^{241} \mathrm{Am}$, which emits a $59.5 \mathrm{keV} \gamma$, was used as a calibration source. 


\subsection{The CRESST Test Cryostat}

The prepared crystal and sources were mounted in the CRESST Test Cryostat. The base temperature of the ${ }^{3} \mathrm{He} /{ }^{4} \mathrm{He}$-dilution refrigerator is $7 \mathrm{mK}$ [3].

The cryogenic calorimeters used in this measurement are highly sensitive to mechanical vibrations from the cryostat pumps. To minimize noise from these vibrations, the detector mount is suspended from a specially-machined bronze spring. This suppresses all vibrations above a few Hertz, leaving only vibrations that to not contribute to detector noise.

Once the detector mount was wired and the radiation shields and cryostats were sealed, the cryostat was cooled to $\mathscr{O}(10 \mathrm{mK})$.

\subsection{Data Acquisition}

Current changes in the two TES are measured using SQUID (Superconducting Quantum Interference Device) amplifiers. The trigger is set to begin data recording on pulse onset in either channel. One bias supply chain provides current across the TES, and a separate chain powers the heater.

To stabilize the TES temperature, a "control pulse" of the appropriate amplitude to drive the TES out of the transition region is sent through the heater every six seconds. For these pulses, the amplitude from the SQUID reflects the difference between the operating point and the top of the transition region. The operating point is kept stable by varying the heater bias to keep this amplitude constant. The heater is also used to inject a sequence of test pulses of up to twelve different amplitudes at 30 second intervals. This allows the calibration to be extended over the full dynamic range of the detector unit.

\subsection{Tuning the TES}

Before data can be taken, the operating temperature and current of each TES and the amplitude of the control pulses from the heater must be set. This is done via "heater sweeps" like those seen in Figure 2a. The bias across each TES should be high enough to maximize pulse amplitude, but low enough to avoid self-heating effects, which broaden the transition and decrease sensitivity. Once a bias current is chosen, the operating temperature of the TES is chosen. This choice can have a significant impact on detector performance because the TES transition region is imperfect- its slope, which determines the sensitivity and linear response regime of the detector, can vary. The operating point is chosen so that the length of the linear slope region above the point is maximized, which maximizes the linear response regime of the detector. The point is also chosen to use the highest possible slope region of the transition, to maximize sensitivity. Finally, the size of the control pulse and its expected amplitude are determined from the same heater sweep curve.

For this data run, a bias of $9 \mu \mathrm{A}$ was chosen for the phonon detector Sabine, and a bias of $2 \mu \mathrm{A}$ was chosen for the photon detector Pierre. The set point of the heater was chosen to be $.45 \mathrm{~V}$ for Sabine, and the set point for Pierre was chosen to be 3.2 V. See Figure $2 b$.

\section{Data Analysis}

\subsection{Template Fit}

To determine pulse amplitudes, a correlated truncated template fit is used. A template for each 


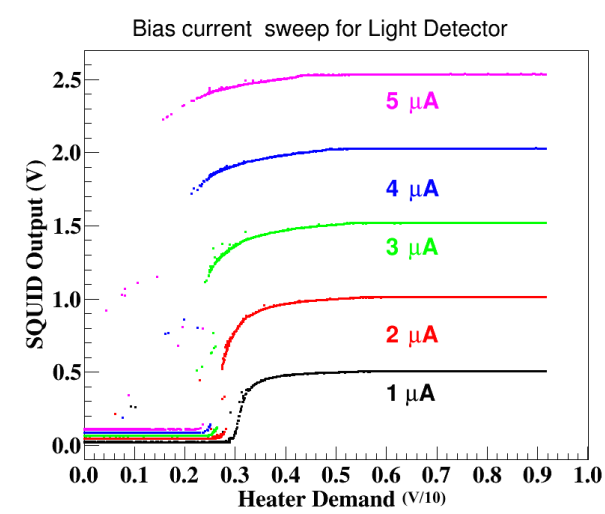

(a) The heater sweeps done to find the appropriate TES operating current. A current of $2 \mu \mathrm{A}$ was chosen.

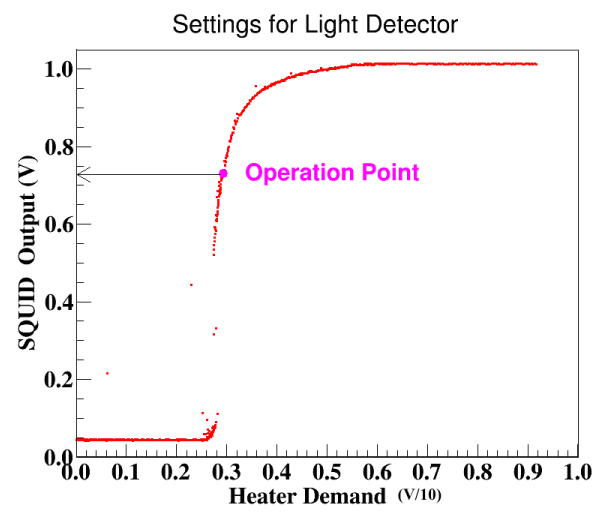

(b) The heater set point for Pierre was chosen to be $3.2 \mathrm{~V}$. The expected amplitude of control pulses can also be taken from this plot.

Figure 2: The measurements taken to tune the TES for Pierre, the photon detector. Similar measurements were taken for Sabine, the phonon detector.

detector is made by averaging the pulses from a low energy calibration source, in this case the 59.5 $\mathrm{keV} \gamma$ from the ${ }^{241} \mathrm{Am}$ source. The averaging removes much of the noise. Pulses with significant baseline tilt, indicating pileup, or where one of the channels failed to record a pulse, indicating a direct hit to the TES or photon detector, are not used for the template.

All the pulses are then fitted with the template, allowing the template pulse height, baseline offset, and start time offset to vary. A correlated fit is used to help determine the relative pulse onset times in the two channels. This improves sensitivity to low-amplitude pulses in the photon channel [3].

Pulses exceeding the linear range of the transition will have suppressed amplitudes and a different shape than low-energy pulses. Therefore, a truncated template fit should be used for these pulses. The truncation point is chosen to be the maximum amplitude for which the RMS of the template fit is no longer constant. The template fit is performed again, with truncation implemented. See Figure 3a. This truncated fit gives the amplitude values that would be seen for these pulses if the transition region were linear in the appropriate range. Therefore, the values found from this fit correlate with energy.

A final cut on the RMS of the fit removes pileup, particularly noisy events, direct hits, and other unwanted events.

\subsection{Energy Calibration}

Using the $59.5 \mathrm{keV} \gamma$ peak and the test pulses from the heater, the two detectors are calibrated. An energy of $59.5 \mathrm{keV}$ is assigned to the fitted peak value, and the calibration function is determined from a low-order polynomial fit to the heater pulse amplitudes. This gives a calibration that can be used over the full energy range of the detectors. For the photon detector, the energies found are in $\mathrm{keV}_{e e}$ (electron equivalent), indicating that the light yield for electron recoils in the calibration peak has been normalized to one. 


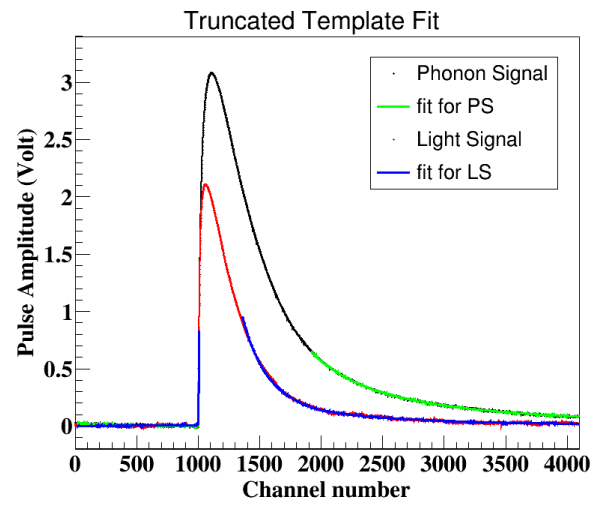

(a) Large amplitude pulses are fit with a truncated template fit.

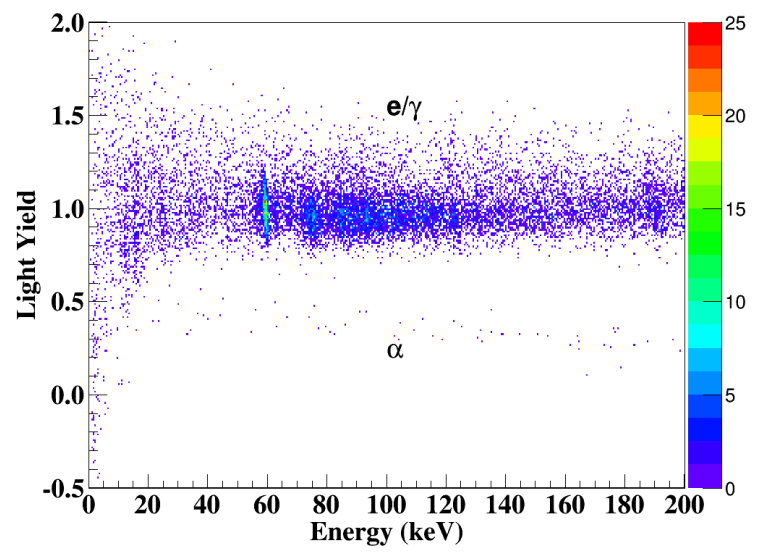

(b) The low energy region shows that $\alpha$ particle light yield is energy-dependent. Preliminary data.

Figure 3: A truncated template fit is used to determine pulse amplitudes. Data are plotted in light yield (the ratio of photon energy to phonon energy, with the ${ }^{241} \mathrm{Am} 59.5 \mathrm{keV}$ peak ratio normalized to one) vs. phonon energy.

\section{Results and Discussion}

The preliminary data, taken during the LNGS Summer Institute, show that the setup used is appropriate for studying the light yield of $\alpha$ scattering events. Even with only a few days of datataking and without optimizing the analysis used, the alpha band is clearly visible at all energies. The light yield at low energies, in Figure 3b, shows that the light yield of $\alpha$ particles increases at low energies.

A complete data set, to be published, will allow a precise fit to the $\alpha$ band, determining its energy dependence in this and other crystals measured. This will potentially allow a re-analysis of CRESST-II data that sets stronger limits on the presence of WIMPs.

\section{Acknowledgments}

Our thanks go to Karoline Schäffner for sharing her work, knowledge, and time with us, and to LNGS and the Summer Institute organizing committee for putting together a wonderful program of lectures and hands-on work. This material is based upon work supported by the National Science Foundation Graduate Research Fellowship Program under Grant No. DGE 1256082.

\section{References}

[1] G. Angloher et. al., Results from 730 kg dats of the CRESST-II Dark Matter Search EPJC 721971 (2012) [astro-ph.co/1109.0702].

[2] CRESST Collaboration, Results on low mass WIMPs using an upgraded CRESST-II detector EPJC 74 (12), 3184 (2014) [astro-ph. CO/1407.3146].

[3] K. J. Schäffner, "Study of Backgrounds in the CRESST Dark Matter Search" Ph.D. dissertation, Max Planck Inst. Phys., Werner Heisenberg Inst., Munich, DEU. 